Check for updates

Cite this: J. Mater. Chem. B, 2020 8,578

DOI: $10.1039 / c 9 t b 90179 e$

rsc.li/materials-b

\section{Correction: A fast-dissolving microneedle array loaded with chitosan nanoparticles to evoke systemic immune responses in mice}

\author{
Zhilin Li, ${ }^{a}$ Yingju He, ${ }^{a}$ Li Deng, $^{{ }^{a}}$ Zhi-Rong Zhang ${ }^{a}$ and Yunzhu Lin ${ }^{b}$
}

Correction for 'A fast-dissolving microneedle array loaded with chitosan nanoparticles to evoke systemic immune responses in mice' by Zhilin Li et al., J. Mater. Chem. B, 2020, DOI: 10.1039/c9tb02061f.

The $x$-axis labels in Fig. 6c were reported incorrectly in the original manuscript. In the original manuscript they were written as "SC free OVA" and "MN free OVA", however the correct labels are "SC free OVA + CpG" and "MN free OVA + CpG", as shown in the corrected figure below:

a

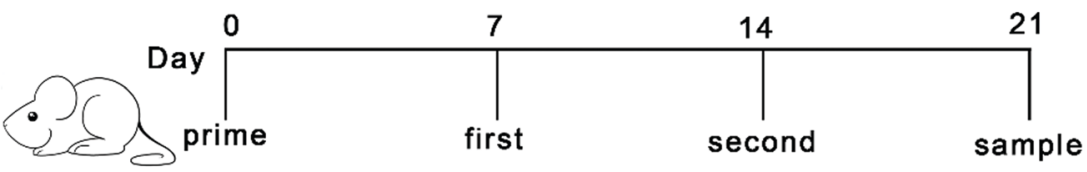

b
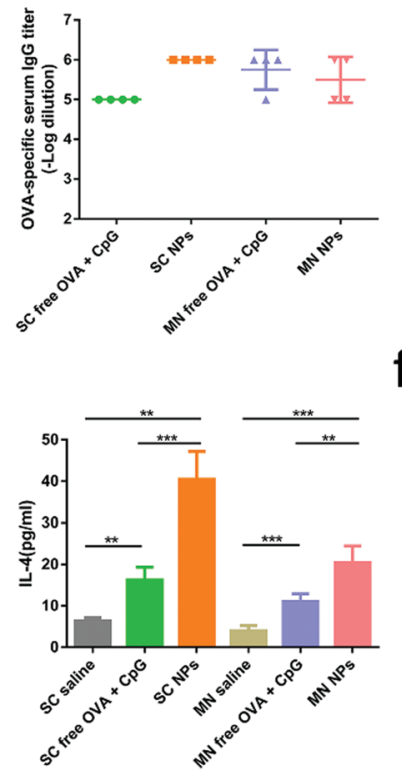

C

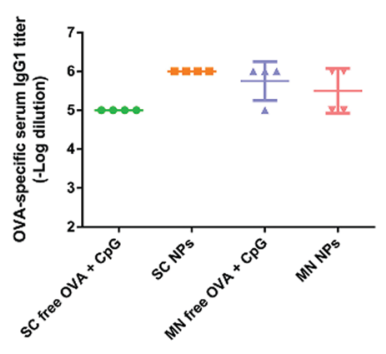

f

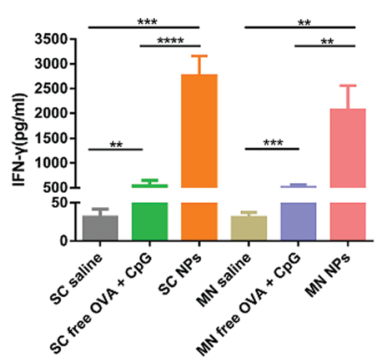

d

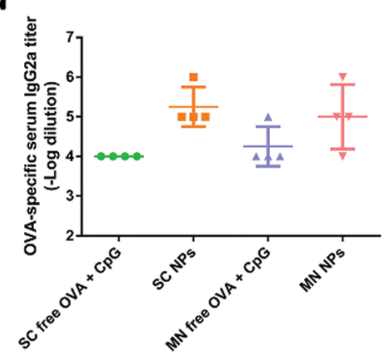

9

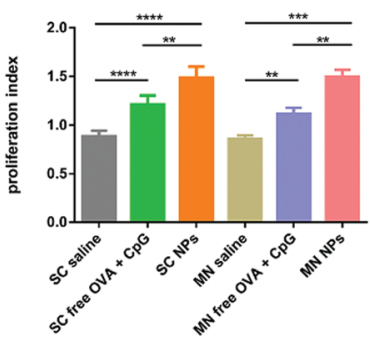

Fig. 6 CS-OVA-CpG NPs induce effectively immune responses. (a) 6-8-week-old female BALB/c mice were immunized with various formulations three times at one-week intervals and the blood and spleen samples were collected on day 21 to evaluate the humoral and cellular responses. Antigen specific titers of IgG (b), IgG1 (c) and IgG2a (d) were determined using ELISA kits. Cytokines IL-4 (e) and IFN- $\gamma$ (f) secreted by culturing splenocytes collected from the vaccinated mice. (g) Proliferation index of the splenocytes obtained from the vaccinated mice. Data represent means $\pm \operatorname{SD}(n=5) .{ }^{* \star} p<0.01,{ }^{* \star *} p<0.001,{ }^{* \star * *} p<0.0001$.

\footnotetext{
${ }^{a}$ Key Laboratory of Drug-Targeting and Drug Delivery System of the Education Ministry and Sichuan Province, Sichuan Engineering Laboratory for Plant-Sourced Drug and Sichuan Research Center for Drug Precision Industrial Technology, West China School of Pharmacy, Sichuan University, Chengdu 610041, China.

E-mail: denglisunny@163.com

${ }^{b}$ Department of Pharmacy, West China Second University Hospital and Evidence-Based Pharmacy Center and Key Laboratory of Birth Defects and Related Diseases of Woman and Children, Sichuan University, Chengdu 610041, China
} 
The term "Free OVA + CpG" in the legend of Fig. 4a and b should be corrected to "Free OVA". The term " *** $p<0.001$ " in the figure legend should also be corrected to "****p $<0.0001$ ". Please see the corrected figure and figure legend below.
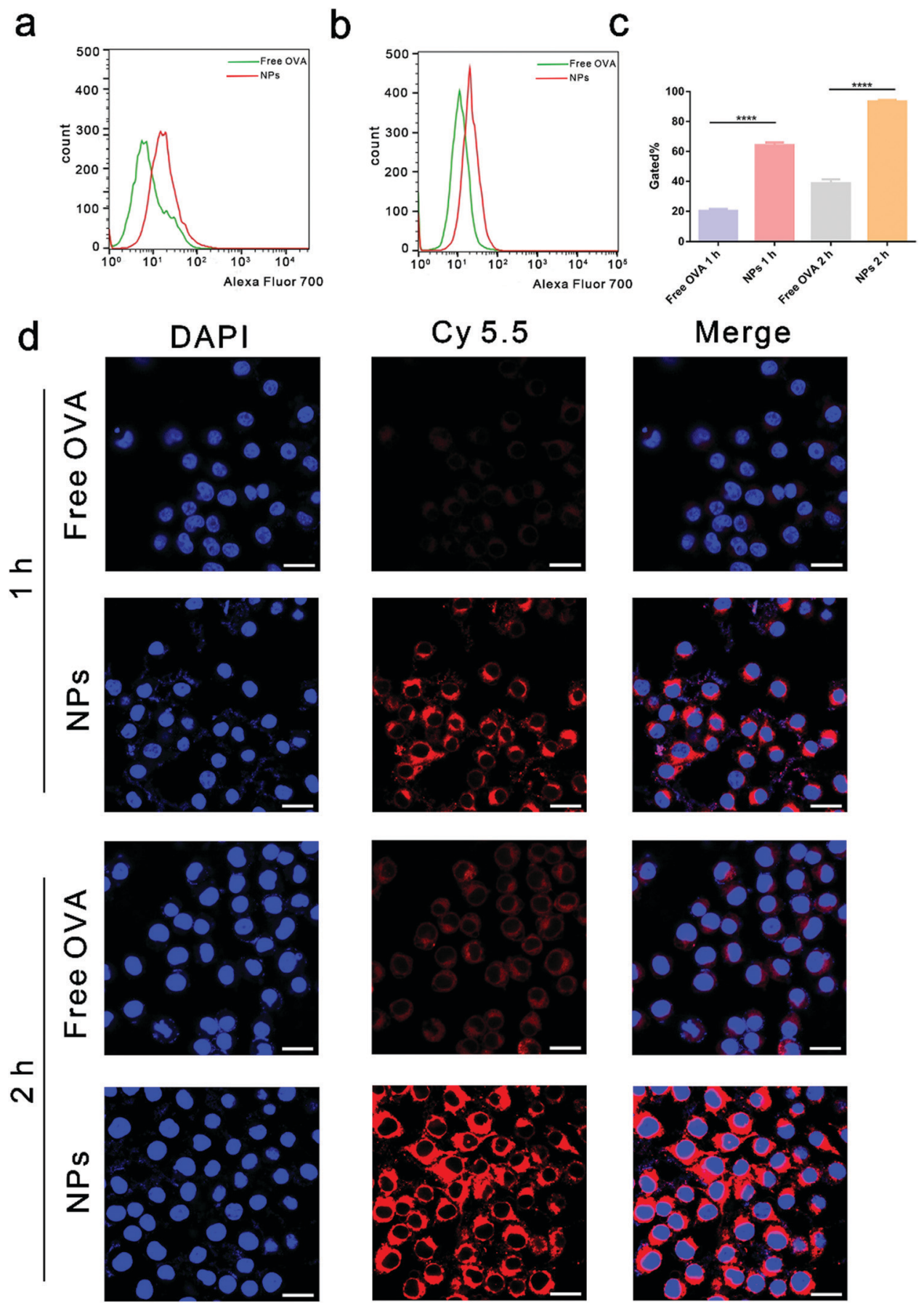

Fig. 4 CS-OVA-CpG NPs promoted internalization of OVA into DC2.4 cells. Cells were incubated with free Cy5.5-OVA and CS-OVA-CpG NPs for $1 \mathrm{~h}$ (a) and $2 \mathrm{~h}$ (b) at $37{ }^{\circ} \mathrm{C}$ using flow cytometry to evaluate the uptake efficiency. (c) Uptake efficiency of CS-OVA-CpG NPs in DC2.4 cells. (d) Representative confocal laser scanning microscopy images of DC2.4 cells incubated for 1 and $2 \mathrm{~h}$ with CS-OVA-CpG NPs (red, Cy5.5-OVA NPs; blue, cell nuclei). Scale bars represent $20 \mu \mathrm{m} .{ }^{* \star * \star} p<0.0001$.

The Royal Society of Chemistry apologises for these errors and any consequent inconvenience to authors and readers. 